\title{
Mitochondrial genomes organization in alloplasmic lines of sunflower (Helianthus annuus) with various types of cytoplasmic male sterility
}

\author{
Maksim S. Makarenko ${ }^{\text {Corresp., }}{ }^{1}$ ， Igor V. Kornienko ${ }^{1,2}$ ， Kirill V. Azarin ${ }^{1}$ ， Alexander V. Usatov ${ }^{1}$, Maria D. \\ Logacheva $^{3}$, Nicolay V. Markin ${ }^{1}$, Vera A. Gavrilova ${ }^{4}$ \\ ${ }^{1}$ Southern Federal University, Rostov-on-Don, Russia \\ 2 Southern scientific center of the Russian Academy of Sciences, Rostov-on-Don, Russia \\ 3 Moscow State University, Belozersky Institute of Physical and Chemical Biology, Moscow, Russia \\ ${ }^{4}$ The N.I. Vavilov All Russian Institute of Plant Genetic Resources, Saint Petersburg, Russia \\ Corresponding Author: Maksim S. Makarenko \\ Email address: mcmakarenko@yandex.ru
}

Background. Cytoplasmic male sterility (CMS) is a common phenotype in higher plants, which often is associated with rearrangements in mitochondrial DNA (mtDNA), and is widely used to produce hybrid seeds in a variety of valuable crop species. The CMS phenomenon investigations are also promote understanding of a fundamental issue of nuclear-cytoplasmic interactions in the ontogeny of higher plants. In the present study, we analyzed the structural changes in mitochondrial genomes of three alloplasmic lines of sunflower (Helianthus annuus). The investigation was focused on CMS line PET2, as there are very few reports about its mtDNA organization.

Methods. The NGS sequencing, de novo assembly, and annotation of sunflower mitochondrial genomes were performed. The comparative analysis of mtDNA of HA89 fertile line and two HA89 CMS lines (PET1, PET2) occurred.

Results. The mtDNA of the HA89 fertile line was almost identical to the HA412 line (NC_023337). The comparative analysis of HA89 fertile and CMS (PET1) analog mitochondrial genomes revealed $11852 \mathrm{bp}$ inversion, 4732 bp insertion, 451 bp deletion and 18 variant sites. In mtDNA of HA89 (PET2) CMS line 77 kb translocation, 711 bp and 3780 bp deletions, as well as 1558 bp, 5050 bp, 14330 bp insertions were determined. There are also revealed 83 polymorphic sites sites in the PET2 mitochondrial genome, as compared with the fertile line

Discussion. Among the revealed rearrangements the $1558 \mathrm{bp}$ insertion resulted in new open reading frames formation - orf228 and orf246. The orf228 and orf246 could be the main reason for the development of PET2 CMS phenotype, whereas the role of other mtDNA reorganizations in CMS formation is negligible. 
1 Mitochondrial genomes organization in alloplasmic lines of sunflower (Helianthus annuus)

2 with various types of cytoplasmic male sterility

3

4 5

6

Maksim S. Makarenko $^{1}$, Igor V. Kornienko ${ }^{1,2}$, Kirill V. Azarin ${ }^{1}$, Alexander V. Usatov ${ }^{1}$, Maria D. Logacheva $^{3}$, Nikolay V. Markin 1 , Vera A. Gavrilova ${ }^{4}$

${ }^{1}$ Southern Federal University, Rostov-on-Don, Russia

${ }^{2}$ Southern scientific center of the Russian Academy of Sciences, Rostov-on-Don, Russia

${ }^{3}$ Lomonosov Moscow State University, Belozersky Institute of Physical and Chemical Biology, Moscow, Russia

${ }^{4}$ The N.I. Vavilov All Russian Institute of Plant Genetic Resources, Saint Petersburg, Russia Corresponding Author: Maksim Makarenko Email address: mcmakarenko@yandex.ru

\section{Abstract}

Background. Cytoplasmic male sterility (CMS) is a common phenotype in higher plants, that is often associated with rearrangements in mitochondrial DNA (mtDNA), and is widely used to produce hybrid seeds in a variety of valuable crop species. Investigation of the CMS phenomenon promotes understanding of fundamental issues of nuclear-cytoplasmic interactions in the ontogeny of higher plants. In the present study, we analyzed the structural changes in mitochondrial genomes of three alloplasmic lines of sunflower (Helianthus annuus). The investigation was focused on CMS line PET2, as there are very few reports about its mtDNA organization.

Methods. The NGS sequencing, de novo assembly, and annotation of sunflower mitochondrial genomes were performed. The comparative analysis of mtDNA of HA89 fertile line and two HA89 CMS lines (PET1, PET2) occurred.

Results. The mtDNA of the HA89 fertile line was almost identical to the HA412 line (NC_023337). The comparative analysis of HA89 fertile and CMS (PET1) analog mitochondrial genomes revealed 11852 bp inversion, 4732 bp insertion, 451 bp deletion and 18 variant sites. In the mtDNA of HA89 (PET2) CMS line we determined 27,5 kb and 106,5 kb translocations, 711 
32 bp and $3780 \mathrm{bp}$ deletions, as well as, $5050 \mathrm{bp}$ and $15885 \mathrm{bp}$ insertions. There are also 83 33 polymorphic sites in the PET2 mitochondrial genome, as compared with the fertile line.

\section{Discussion.}

The observed mitochondrial reorganizations in PET1 resulted in only one new open reading frame formation (orfH522), and PET2 mtDNA rearrangements led to the elimination of orf777, duplication of atp6 gene and appearance of four new ORFs with transcription activity specific for the HA89 (PET2) CMS line - orf645, orf2565, orf228 and orf285. Orf228 and orf285 are the atp 9 chimeric ORFs, containing transmembrane domains and possibly may impact on mitochondrial membrane potential. So orf228 and $\operatorname{orf} 285$ may be the cause for the appearance of the PET2 CMS phenotype, while the contribution of other mtDNA reorganizations in CMS formation is negligible.

\section{Introduction}

In plants, the phenomenon of cytoplasmic male sterility (CMS) stems from interaction between mitochondrial and nuclear genomes resulting in microsporogenesis disorders (Touzet \& Meyer, 2014). All known natural CMSs, as well as most of the artificially obtained examples, are characterized by a special type of mitochondrial DNA (mtDNA) with numerous structural rearrangements as compared to the mtDNA of the fertile plants of the same species (Ivanov \& Dymshits, 2007; Horn, Gupta \& Colombo, 2014; Garayalde et al. 2015). The mitochondrial genomes of higher plants have comparatively large sizes with a multitude of noncoding and repetitive sequences that can result in the complex of sub-genomic structures (Chen \& Liu, 2014). These features of plant mtDNA promote a large number of recombination events, leading to the appearance of new sequences and new open reading frames, that in turn often results in CMS development (Horn, Gupta \& Colombo, 2014; Touzet \& Meyer, 2014).

Natural CMS forms have been described in more than 150 species of flowering plants (Fujii \& Toriyama, 2009). Most CMS sources in crops are obtained using interspecific hybridization. The first CMS in a sunflower was discovered by Leclercq (1969) in an interspecific hybrid between Helianthus petiolaris Nutt (PET1) and Helianthus annuus. Comparison of mitochondrial DNA organization of the fertile line and the male-sterile line carrying the PET1 cytoplasm revealed the presence of an 11-kb-inversion and 5-kb-insertion 
63 (Siculella \& Palmer, 1988; Kohler et al., 1991). These rearrangements of the mitochondrial 64 genome produced a new open reading frame (orfH522) in the 3'-flanking region of the atpl gene encoding the alpha subunit of mitochondrial F1 ATPase. A new orfH522 is co-transcribed with the atpl gene as a polycistronic mRNA (Moneger, Smart \& Leaver, 1994). Using antibodies specific to the product of orfH522 gene (16-kDa-protein), Horn et al. (1996) showed that this was the only difference between the mitochondrial translation products of fertile and CMS lines. The $16-\mathrm{kDa}$ protein is synthesized in all tissues of a plant. It is embedded in the mitochondrial membranes and is believed to disrupt its integrity (Horn et al., 1996). Expression of orfH522 in tapetum cells leads to premature apoptosis. Release of cytochrome $\mathrm{C}$ from the mitochondria, activates the proteolytic enzyme cascade, eventually leading to degradation of nuclear DNA and cell death (Balk \& Leaver, 2001; Sabar et al., 2003). Interestingly, stable transgenic CMS tobacco lines carrying the orfH522 gene were obtained (Nizampatnam et al., 2009). When dominant nuclear restorer gene (Rf) is present in the sunflower genome, fertility is restored due to the anther-specific lowering of the co-transcript of orfH522 and the atpl gene (Moneger, Smart \& Leaver, 1994; Horn, 2003). A possible mechanism leading to a reduction in the number of the chimeric atp1-orfH522 transcripts by the restorer gene is polyadenylation of RNA matrices, causing accelerated degradation of RNA molecules by the ribonuclease (Gagliardi \& Leaver, 1999).

The CMS-Rf system is widely used for the commercial production of F1 hybrid seeds for many important crops, including maize, sorghum, sunflower etc. (Liu et al., 2011, Bohra et al., 2016). Almost all commercial sunflower hybrids are currently based on a single source of CMS discovered by Leclercq (1969) and described above. Such genetic homogeneity of cultivated hybrids makes them extremely vulnerable to new virulent strains of the pathogens and can lead to negative phenomena, for example, epiphytotics development (Levings, 1990). For instance, leaf blight pandemic affected only one type maize hybrids (namely, Texas-type), while other types of CMS were less susceptible to this disease (Bruns, 2017). To create new CMS-Rf systems, prevent mtDNA unification, and reduce genetic vulnerability of sunflower hybrids to biotic and abiotic stresses, it is urgent to search for and introduce the new CMS sources into sunflower breeding. Although more than 70 cytoplasmic male sterility types have been identified in sunflower (Garayalde et al. 2015), they have not been sufficiently studied, resulting in limitation of their use in commercial hybrid breeding. Undoubtedly, research of the cytoplasmic 
94

95

96

97

male sterility phenomenon is important for investigating the fundamental problem of nuclearcytoplasmic interaction in the ontogeny of higher plants (Hanson \& Bentolila, 2004). Previously, the comparison of mitochondrial genome organization between $28 \mathrm{CMS}$ sources of sunflower, performed with Southern hybridization, demonstrated that some types of CMS (for example, ANN2, PET2, PEF1, etc.) have a different organization of the mtDNA from the PET1-like cytoplasms (Horn, 2002). Sequencing and comparing of whole mitochondrial genomes of various CMS sources will provide additional information about the molecular changes in their mtDNA, which in turn could help to suggest new mechanisms of the male sterility formation. In the current study, we investigated structural changes in mitochondrial genomes of HA89-alloplasmic lines: fertile line and two analog lines with different types of cytoplasmic male sterility - PET1 and PET2. The results obtained for the PET2 CMS type formed the basis for further research.

\section{Materials and methods}

\section{Plant material}

Fertile line HA89 and isonuclear CMS lines - PET1 and PET2 of sunflower were obtained from the genetic collection of the N. I. Vavilov Institute of Plant Genetic Resources (VIR, Russia). The lines had the same nuclear genome (HA89), but they differed in chloroplast and mitochondrial genomes, inherited from their wild ancestors. The CMS sources were initially obtained by the interspecific hybridization of domesticated sunflower (Helianthus annuus) with H. petiolaris Nutt (Leclercq, 1969; Whelan, 1980).

\section{Mitochondrial DNA extraction, genome library construction and NGS sequencing}

We extracted the organelle fraction with a reduced amount of nuclear DNA from leaves of 14-day sunflower seedlings, following the protocol of Makarenko et al. (2016). For every line, we used the same quantity of leaf tissue from five plants. The DNA isolation was performed with PhytoSorb kit (Syntol, Russia), according to the manufacturer's protocol. The NGS libraries preparations were made using 1 ng of DNA and Nextera XT DNA Library Prep Kit (Illumina, USA), following the sample preparation protocol by Illumina. For the qualitative control of libraries, Bioanalyzer 2100 (Agilent, USA) was used. The libraries quantitation was performed with the Qubit fluorimeter (Invitrogen, USA) and qPCR. Libraries for NGS sequencing were diluted up to the concentration of $8 \mathrm{pM}$. Libraries were sequenced on different sequencing 
124 platforms. Fertile line and PET1 NGS libraries were sequenced with NextSeq 500 sequencer 125 using High Output v2 kit (Illumina, USA). A total number of 13,240,057 150-bp paired reads 126 were generated for fertile line and 14,758,067 reads - for PET1 line. PET2 library was sequenced 127 with HiSeq2000 and MiSeq platforms using TruSeq SBS Kit v3-HS and MiSeq Reagent Kit v2 128 500-cycles (Illumina, USA). A total number of 4,471,774 125-bp and 4,931,318 250-bp paired 129 reads were generated for the PET2 line.

130

131

132

133

134

135

136

137

138

139

140

141

142

143

\section{Analysis of sequencing data}

Quality of reads was determined with Fast QC. Trimming of adapter-derived and low quality (Q-score below 25) reads was performed with Trimmomatic software (Bolger et al., 2014). Using the Bowtie 2 tool v 2.3.3 (Langmead \& Salzberg, 2012) sequencing reads were aligned to the reference sequence from NCBI databank (NC_023337.1). The Bowtie 2 alignments were done only for concordant paired reads (--no-mixed, --no-discordant options). Variant calling was made with samtools/bcftools software (Li, 2011) and manually revised using the IGV tool (Thorvaldsdottir, Robinson \& Mesirov, 2013). De novo assembly was performed with SPAdes Genome Assembler v 3.10.1 (Nurk et al., 2013) with different K values equal to 75, $85,95,127$, and read coverage cutoff value equal to 30.0 (--cov-cutoff option). The potential ORFs were identified using ORFfinder. The graphical genome map was generated using the OGDRAW tool (Lohse, Drechsel \& Bock, 2007). Transmembrane domains were predicted using the TMHMM Server v.2.0 (available online: http://www.cbs.dtu.dk/services/TMHMM-2.0/).

\section{Validation of genome assembly. PCR and Sanger sequencing}

The contigs obtained in de novo assembly were aligned to the reference sunflower mitochondrial genome (NC_023337.1) using BLAST. Validation of discovered rearrangements was made by PCR analysis and Sanger sequencing. PCR reactions were performed with LongAmp Taq PCR Kit (New England BioLabs, USA) for reactions with expected amplicons more than $1.5 \mathrm{~kb}$, and with Tersus Plus PCR kit (Evrogen, Russia) for other reactions, including Sanger sequencing. For 28-29 cycles of PCR, we used $0.4 \mathrm{uM}$ of primers (Table 1) and $1 \mathrm{ng}$ of extracted DNA. The direct sequencing of purified amplicons was performed using the BigDye Terminator v3.1 Cycle Sequencing Kit (Thermo Fisher Scientific, USA) and ABI Prism 3130x1 Genetic Analyser (Applied Biosystems, USA). 
153

154

155

156

157

158

159

160

161

162

163

164

165

166

167

168

169

170

171

172

173

174

175

176

177

178

179

180

181

\section{RNA extraction and $q R T-P C R$}

Total RNA from the leaves of five samples of each line was extracted with guanidinium thiocyanate-phenol-chloroform reagent kit - ExtractRNA (Evrogen, Russia). RNA quality and concentration were measured using the NanoDrop 2000 spectrophotometer (Thermo Fisher Scientific, USA) and the Qubit fluorimeter (Invitrogen, USA). $0.5 \mu \mathrm{g}$ of total RNA was treated with DNAse I (Thermo Fisher Scientific, USA) according to the manufacturer's instruction. First-strand cDNA was synthesized using MMLV RT kit (Evrogen, Russia) and specific primers. The qPCR was performed with designed primers (Table 1) and PCR kit with EvaGreen dye (Syntol, Russia) on Rotor-Gene 6000 (Corbett Research, Australia).

\section{Results and Discussion}

\section{Organization of fertile line and PET1 mtDNA}

De novo assembly of the fertile line and PET1 mitochondrial genomes revealed between 9 and 12 large contigs (10-115 kb long), depending on $\mathrm{K}$ value. The optimum $\mathrm{K}$ value was 95 .

The obtained large contigs covered up to $95 \%$ of the reference genome. The remaining $5 \%$ of the mitochondrial genome correspond to repeats, therefore they were the breakpoints of contigs formation. Among numerous repeats in the mitochondrial genome, only one large $(12,933 \mathrm{bp}$ long) and six small (203-728 bp long) repeats played a crucial role in genome assembly and prevented single scaffold formation. Four regions (415-1192 bp long) with 99\% chloroplast DNA identity also affected the mitochondrial genome assembly. Eventually, manual assembly based on predominant contigs obtained by SPAdes supplemented by analysis of reads alignment by Bowtie 2 and validation of controversial regions performed by PCR analysis and Sanger sequencing, allowed summary sequencing of data in completed mitochondrial genomes. Circular mtDNA of HA89 and PET1 lines are presented in Figures 1 and 2.

The mtDNA comparative analysis of sunflower fertile lines HA412 (NCBI accession NC_023337.1) and HA89 revealed two single nucleotide thymidine insertions: in positions 35690-35691 and 129368-129369 of NC_023337. Two SNP in the noncoding part of $301 \mathrm{~kb}$ genome is a negligible difference. We did not amend the HA89 mitochondrion sequence in the NCBI GenBank and, in this paper, used the same positions of mtDNA for HA412 and HA89 lines for simplicity. 
182

183

184

185

186

187

188

189

190

191

192

193

194

195

196

197

198

199

200

201

202

203

204

205

206

207

208

209

210

211

212

The PET1 mitochondrial genome had structural rearrangements as well as polymorphic sites compared to the fertile lines' (HA412/HA89) mtDNA. Previously, using the restriction analysis and Sanger sequencing, large structural variations of mtDNA associated with the sterility of plants were detected in the PET1 CMS type of sunflower - a $11 \mathrm{~kb}$ inversion and a 5 kb insertion (Kohler et al., 1991). The results of the current study not only confirmed the presence of these reorganizations in the HA89 PET1 mitochondrial genome but also allowed detection of more precise genome changes: 11852 bp inversion, 4732 bp insertion, 451 bp deletion. The revealed insertion was $98 \%$ identical to the PET1 insertion that can be obtained from GenBank NCBI (accession Z23137.1). Comparing with the fertile line genome, we have identified 18 variants in HA89(PET1) mtDNA. Among the nucleotide variations, 8 were localized in SSR loci, 2 deletions (single and dinucleotide) and 7 SNP, including 1 transition and 6 transversions, were predominantly located in noncoding regions (Table 2). The exceptions were nonsynonymous mutations in orf777 (Asp251Glu), nad6 (Ser232Tyr), rpl16 (Lys32Gln). The HA89 (PET1) complete mitochondrial genome sequence has been deposited in the NCBI databank (accession MG735191).

\section{Organization of PET2 mtDNA}

We assembled the PET2 mitochondrial genome following the described procedure that was used for identification of HA89 fertile and PET1 complete mtDNA sequences. The complete mitochondrion of HA89(PET2) is 316586 bp (Figure 3) and, in comparison with the HA89 fertile line, contained large-scale reorganizations of the mtDNA structure as well as minor changes represented by variable sites. Among significant rearrangements, two translocations, two deletions and two insertions were determined.

Even in a single plant cell, the mitochondrial genome is represented by several DNA molecules with various structure (Sloan et al., 2012). The so-called "master chromosome" - the single mtDNA molecule is a rare type of mitochondrial genome organization (Gualberto et al., 2014). More often, a mitochondrion includes a set of sub-genomic forms (Yang et al., 2015).

Because sub-genomes could form differing master chromosomes, the statement of translocations in plant mitochondrial genomes is equivocal. To compare complete mitochondrial genomes of HA89(PET2) and fertile HA89 lines, two translocations of approximately 27,5 kb and 106,5 kb (positions 37112-64614 bp and 194439-300945 bp in HA89 fertile line mtDNA) could be established. Using specific PCR primers (table 1) we could demonstrate that the mtDNA in 
213 sunflower can also form a 154-kb sub-genomic circle molecule (positions 36393-190650). In the

214 sunflower genome there is a repeat region of $722 \mathrm{bp}(36393-37114=189929-190650$ positions

215 of the fertile line) with $100 \%$ similarity, which makes the cyclization of sub-genome circular

216 molecule possible. In the case of HA89(PET2) this sub-genome circle molecule has the wrong

217 insertion point in the genome as compared with the fertile analog and thus results in

218 translocations appearance.

219 Deletion of $711 \mathrm{bp}(35682-36393$ positions in fertile line mtDNA) resulted in the absence 220 of orf777 in the HA89(PET2) mitochondrial genome. The orf777 codes for a putative protein 221 with unknown function, which shows no similarities with other mitochondrial proteins. So its 222 elimination can hardly be the molecular reason for CMS development. The other deletion of 2233780 nucleotides (190659-194439 positions) affects only noncoding sequences. Notably, the 224 deletions are associated with sub-genome integration regions (36393-37114 and 189929-190650 225 positions). So there is a possibility, that deletions in these regions could impair master chromosome assembly, which results in the translocations formations, as described above.

More significant mtDNA reorganizations are two revealed insertions - $5050 \mathrm{bp}$ and $15885 \mathrm{bp}$. Among them, the $5050 \mathrm{bp}$ insertion is most likely not involved in the origin of the CMS phenotype. This insertion was found in the intergenic region atp6-cox2 (275230-275231 positions of fertile line mtDNA), and it does not lead to the formation of new open reading frames (ORFs) directly in the place of insertion into the mitochondrial genome. Moreover, there are no large (more than 300 nucleotides) ORFs within the sequence of $5050 \mathrm{bp}$ insertion. The exception is orf645 putatively translated into a polypeptide of 215 amino acids. Twenty-three amino acids at the N-terminus of this 215 amino acid protein were similar to N-terminus of ribosomal protein S3. We determined transcripts of orf645 in PET2 CMS line by qPCR, using specific primers (Table 1). However, mRNA of orf645 was absent in the fertile line and PET1 CMS line. Most often the molecular reason for CMS phenotype development is the emergence of chimeric ORFs with transmembrane domains, such as ATPase complex subunits, respiratoryrelated proteins, etc. (Gillman, Bentolila \& Hanson, 2007; Yang, Huai \& Zhang, 2009). Consequently, even if orf645 is translated in vivo, its role in male sterility development most likely is negligible, as there are no similarities with respiratory/ATP synthesis-related proteins. 
244 of the insertion (9482 bp) has 100\% similarity to another PET2 mtDNA region (126260-135741

245 positions). The repeating part of the insertion could be divided into two parts - 6097 bp (35686-

24641782 positions of PET2 mitochondrion) are common (99-100\% identity) for Helianthus

247 mitochondrion: 269147-275243, 273418-279514 and 126260-132356 positions of fertile line,

248 PET1 and PET2 CMS lines, respectively. Such repeat predominantly consists of noncoding

249 sequence, except atp6 gene. The other 3385 bp (41783-45167 positions of PET2 mitochondrion)

250 have $100 \%$ similarity to the part of $5050 \mathrm{bp}$ insertion (132357-135741 positions of PET2

251 mitochondrion). However, this part of the insertion does not contain coding sequence. The next

252 part of the 15885 bp insertion counts 4849 nucleotides which are unique for the PET2

253 mitochondrion (45168-50016 positions). The is encoded in this part of the insertion and we

254 determined $\operatorname{orf} 2565$. The last $1554 \mathrm{bp}$ of the $15886 \mathrm{bp}$ insertion (50017-51570 positions of PET2

255 mitochondrion) have complex origin. 390 nucleotides of this insertion (50017-50406 positions of

256 PET2 mitochondrion) are similar to $114179-114568$ positions of fertile line mtDNA, the next

$257271 \mathrm{bp}$ (50406-50676 positions) are unique, then $500 \mathrm{bp}$ (50677-51177 positions) are

258 complement to $114587-115087$ positions of the HA89 fertile mitochondrion and the rest 393

259 nucleotides (50677-51570) are also unique. From a functional point this region presents

260 duplication of atp 9 gene combined with $271 \mathrm{bp}$ insertion and deletion of $12 \mathrm{bp}$, which resulted in

261 two new ORFs formation - orf228 and orf285 (Reddemann, Horn 2018). So the 15885 bp

262 insertion, especially its last 1554 nucleotides, is the most important mitochondrial genome

263 rearrangement, probably associated with PET2 CMS phenotype. It is also notable that $15885 \mathrm{bp}$

264 insertion has the same region in mtDNA of PET2 as the other reorganizations - $711 \mathrm{bp}$ and 3780

265 bp deletions and 106,5 $\mathrm{kb}$ translocation.

266 Summarizing the functional changes data obtained, the $15885 \mathrm{bp}$ insertion consists of

267 four coding sequences: duplicated atp6 gene and three new ORFs - orf 2565 , orf 228 and $\operatorname{orf} 285$.

268 Atp6 chimeric ORFs or new ORFs co-transcribed with atp6 are the quite common causes of

269 CMS development in different plant species (Kim, Kang \& Kim, 2007; Jing et al., 2011; Tan et

270 al., 2017). So we proposed that atp6 gene and its colocalized area are of particular interest as the

271 candidate sequence for CMS phenotype development. The annotation of Helianthus annuus

272 mitochondrion (NC_023337.1) presents the atp6 gene coding mRNA which consists of 906

273 nucleotides. But the fact is that in the 5 ' adjoining sequence (150 bp) to atp6 start codon there is

274 the other one initiating codon (ATG). Transcription from this codon results in new mRNA 
275 counting 1056 nucleotides. The elongated transcript could be translated in putative ATP6 protein

276 with additional 50 amino acids, wherein 37 of 50 amino acids are identical with N-terminus of

277 coxI. The similar extension of protein was discovered in another CMS type of sunflower - ANT1

278 (Spassova et al., 1994), but the described protein had additional 87 aa at the C-terminus of the

279 ATP6 protein. So we assumed that extended transcript, which could be produced in atp6

280 duplicated region, may cause CMS phenotype. To verify the hypothesis, the expression levels of

281 atp6 and 5' elongated atp6 transcripts were analyzed using qPCR with the same reverse primer

282 but different forward primers (Table 1). The elongated atp6 transcript has been expressed at the

283 same level as the atp6 gene (Gene ID: 18250997) both in PET1, PET2 CMS and fertile lines. So

284 the atp6 transcript counting 1056 nucleotides is the normal one, and there is a mistake in its

285 annotation in NC_023337.1. Moreover, the atp9 gene also (Gene ID: 18250970) has wrong

286 transcript annotation - it counts only $261 \mathrm{bp}$ instead of proper one with $300 \mathrm{bp}$. Using qPCR and

287 specific primers (Table 1) we established the same expression level for $261 \mathrm{bp}$ and $300 \mathrm{bp}$

288 transcripts in all three studied lines. Notable that the relative expression level $(\Delta \mathrm{Ct})$ of atp6 gene

289 to atpl gene in PET2 CMS line had no significant difference as compared with fertile and PET1

290 CMS analogs, despite atp6 duplication in PET2 mitochondrion. Thereby atp6 duplication in

291 PET2 mitochondrion does not involve in CMS appearance.

292 All three revealed open reading frames (orf2565, orf228 and $\operatorname{orf} 285)$ had demonstrated

293 transcription activity in PET2 CMS line but not in the fertile or PET1 CMS analogs. The orf2565

294 translates to the putative polypeptide of 855 amino acids. Homology search in the NCBI

295 database using BLAST pointed to similarity to DNA polymerase (type B). The impact of

296 orf2565 on CMS phenotype development is quite doubtful, taking into account that the

297 polypeptide has no transmembrane domains. The orf 228 encodes a polypeptide of 76 aa, of

298 which 75 are identical to C-terminus of ATPase subunit 9, as well as a new start codon (AUG) is

299 formed due to the $271 \mathrm{bp}$ insertion. Naturally, ATPase subunit 9 has two transmembrane

300 domains - near N- and C-terminus, in orf228 there are also two predicted transmembrane

301 domains (Reddemann, Horn 2018). However, it should be noted that the N-terminus

302 transmembrane domain of orf 228 encoded polypeptide lacks four amino acids as compared with

303 atp 9 ones. This difference, in turn, could affect polypeptide (orf228) interaction with

304 mitochondrial membrane and so results in mitochondrial membrane potential changes. The

305 orf 285 encodes a polypeptide of 95 aa, of which 18 are complement to N-terminus of ATPase 
306

307

308

309

310

311

312

313

314

315

316

317

318

319

320

321

322

323

324

325

326

327

328

329

330

331

332

333

334

subunit 9. In total $\operatorname{orf} 228$ and $\operatorname{orf} 285$ polypeptides share 93 of 99 amino acids of the atp 9 protein.

The polypeptide encoded by $\operatorname{orf} 285$ also has the transmembrane domain which formed by predominantly 20-42 aa. Thus both ORFs (orf228, orf285) could be the main reason the development of PET2 CMS type

The comparative analysis of PET2 mitochondrion sequence with complete mtDNA of fertile line revealed 83 polymorphic sites - 14 SSR, 13 small indels (1-29 bp) and 56 SNP (Table 2). Among nucleotide variations only two (synonymous) SNP were in the coding sequence of genes rps3 and atp6. Additional analysis of distribution of variants can offer insight into functional properties and evolution of sunflower mtDNA (Triska et al 2017). Interestingly, PET1 and PET2 share ten polymorphic sites as compared with the fertile line. The obtained HA89(PET2) mitochondrial genome sequence has been deposited to the NCBI GenBank (accessions MG770607.1). It is important to note that the sets of primers used for identification of PET2 insertions may be used for designing molecular markers for this type of CMS in sunflower.

\section{Conclusions}

Comparative analysis of HA89 fertile and PET1 CMS analog mitochondrial genomes revealed 11852 bp inversion, 4732 bp insertion, 451 bp deletion and 18 variant sites. In the mtDNA of HA89 (PET2) CMS line we determined 27,5 kb and 106,5 kb translocations, $711 \mathrm{bp}$ and 3780 bp deletions, as well as, 5050 bp, 15885 bp insertions and 83 polymorphic sites. From a functional point of view, there is the elimination of orf777, duplication of atp6 gene and appearance of four new ORFs with transcription activity specific for the HA89 (PET2) CMS line - orf645, $\operatorname{orf} 2565, \operatorname{orf} 228$ and $\operatorname{orf} 285$. We hypothesize that orf228 and $\operatorname{orf} 285$ could be the main reason for the development of PET2 CMS phenotype, while the contribution of other mtDNA reorganizations in CMS formation is negligible.

\section{References}

1. Balk J, Leaver CJ. 2001. The PET1-CMS mitochondrial mutation in sunflower is associated with premature programmed cell death and cytochrome c release. Plant Cell13:1803-1818. 
335

336

337

338

2. Bohra A, Jha UC, Adhimoolam P, Bisht D, Singh NP. 2016. Cytoplasmic male sterility (CMS) in hybrid breeding in field crops. Plant Cell Reports 35: 967-993. DOI: 10.1007/s00299-016-1949-3.

3. Bolger AM, Lohse M, Usadel B. 2014. Trimmomatic: A flexible trimmer for illumine sequence data. Bioinformatics 30: 2114-2120. DOI: 10.1093/bioinformatics/btu170.

4. Bruns HA. 2017. Southern Corn Leaf Blight: A Story Worth Retelling. Agronomy Journal 109:1218-1224. DOI:10.2134/agronj2017.01.0006.

5. Chen L, Liu YG. 2014. Male sterility and fertility restoration in crops. Annual Review of Plant Biology 65:579-606. DOI:10.1146/annurev-arplant-050213-040119.

6. Fujii S, Toriyama K. 2009. Suppressed expression of RETROGRADE-REGULATED MALE STERILITY restores pollen fertility in cytoplasmic male sterile rice plants. Proceedings of the National Academy of Sciences 106: 9513-9518. DOI: 10.1073/pnas.0901860106.

7. Gagliardi D, Leaver CJ. 1999. Polyadenylation accelerates the degradation of the mitochondrial mRNA associated with cytoplasmic male sterility in sunflower. EMBO Journal 18:3757-66.

8. Garayalde AF, Presotto A, Carrera A, Poverene M, Cantamutto M. 2015. Characterization of a new male sterility source identified in an invasive biotype of Helianthus annuus (L.). Euphytica 206:579-595. DOI:10.1007/s10681-015-1456.

9. Gillman JD, Bentolila S, Hanson MR. 2007. The petunia restorer of fertility protein is part of a large mitochondrial complex that interacts with transcripts of the CMSassociated locus. The Plant Journal 49:217-227. DOI: 10.1111/j.1365313X.2006.02953.x.

10. Gualberto JM, Mileshina D, Wallet C, Niazi AK, Weber-Lotfi F, Dietrich A. 2013. The plant mitochondrial genome: Dynamics and maintenance. Biochimie 100: 107-120. DOI: 10.1016/j.biochi.2013.09.016.

11. Hanson MR, Bentolila S. 2004. Interactions of mitochondrial and nuclear genes that affect male gametophyte development. The Plant Cell 16:154-169. DOI: https://doi.org/10.1105/tpc.015966. 
364

365

366

367

368

369

370

371

372

373

374

375

376

377

378

379

380

381

382

383

384

385

386

387

388

389

390

391

392

393
12. Horn R, Hustedt JE, Horstmeyer A, Hahnen J, Zetsche K, Friedt W. 1996. The CMSassociated $16 \mathrm{kDa}$ protein encoded by orfH522 in the PET1 cytoplasm is also present in other male-sterile cytoplasms of sunflower. Plant Molecular Biology 30:523-538.

13. Horn R. 2002. Molecular diversity of male sterility inducing and male-fertile cytoplasms in the genus Helianthus. Theoretical and Applied Genetics 104:562-570.

14. Horn R, Kusterer B, Lazarescu E, Prüfe M, Friedt W. 2003. Molecular mapping of the Rf1 gene restoring pollen fertility in PET1-based F1 hybrids in sunflower (Helianthus annuus L.). Theoretical and Applied Genetics 106:599-606.

15. Horn R, Gupta KJ, Colombo N. 2014. Mitochondrion role in molecular basis of cytoplasmic male sterility. Mitochondrion 19:198-205. DOI: 10.1016/j.mito.2014.04.004.

16. Ivanov MK, Dymshits GM. 2007. Cytoplasmic male sterility and restoration of pollen fertility in higher plants. Russian Journal of Genetics 43: 354-368. DOI: https://doi.org/10.1134/S1022795407040023.

17. Jing B, Heng S, Tong D, Wan Z, Fu T, Tu J, Ma C, Yi B, Wen J, Shen J. 2012. A male sterility-associated cytotoxic protein ORF288 in Brassica juncea causes aborted pollen development. Journal of Experimental Botany 63: 1285-1295. DOI:10.1093/jxb/err355.

18. Kim DH, Kang JG, Kim BD. 2007. Isolation and characterization of the cytoplasmic male sterility-associated orf456 gene of chili pepper (Capsicum annuum L.). Plant Molecular Biology 63:519-532. DOI 10.1007/s11103-006-9106-y.

19. Köhler RH, Horn R, Lössl A, Zetsche K. 1991. Cytoplasmic male sterility in sunflower is correlated with the co-transcription of a new open reading frame with the atp $A$ gene. Molecular and General Genetics 227:369-376.

20. Langmead B, Salzberg S. 2012. Fast gapped-read alignment with Bowtie 2. Nature Methods 9:357-359. DOI: 10.1038/nmeth.1923.

21. Leclercq P. 1969. Une sterilite male chez le tournesol. Ann Amelior Plant 19:99-106.

22. Levings CS 3rd. 1990. The Texas cytoplasm of maize: cytoplasmic male sterility and disease susceptibility. Science 250:942-947.

23. Li H. 2011. A statistical framework for SNP calling, mutation discovery, association mapping and population genetical parameter estimation from sequencing data.

Bioinformatics 27:2987-2993. DOI: 10.1093/bioinformatics/btr509. 
394

395

396

397

398

399

400

401

402

403

404

405

406

407

408

409

410

411

412

413

414

415

416

417

418

419

420

421

422

423

24. Liu H, Cui P, Zhan K, Lin Q, Zhuo G, Guo X, Ding F, Yang W, Liu D, Hu S, Yu J, Zhang A. 2011. Comparative analysis of mitochondrial genomes between a wheat K-type cytoplasmic male sterility (CMS) line and its maintainer line. BMC Genomics 12:163. DOI: $10.1186 / 1471-2164-12-163$.

25. Lohse M, Drechsel O, Bock R. 2007. OrganellarGenomeDRAW (OGDRAW): a tool for the easy generation of high-quality custom graphical maps of plastid and mitochondrial genomes. Current Genetics 52:267-274. DOI: 10.1007/s00294-007-0161-y.

26. Makarenko MS, Usatov AV, Markin NV, Azarin KV, Gorbachenko OF, Usatov NA. 2016. Comparative Genomics of Domesticated and Wild Sunflower: Complete Chloroplast and Mitochondrial Genomes OnLine Journal of Biological Sciences 16:7175. DOI: 0.3844/ojbsci.2016.71.75.

27. Monéger F, Smart CJ, Leaver CJ. 1994. Nuclear restoration of cytoplasmic male sterility in sunflower is associated with the tissue-specific regulation of a novel mitochondrial gene. EMBO Journal 13:8-17.

28. Nizampatnam NR, Doodhi H, Kalinati Narasimhan Y, Mulpuri S, Viswanathaswamy DK. 2009. Expression of sunflower cytoplasmic male sterility-associated open reading frame, orfH522 induces male sterility in transgenic tobacco plants. Planta 229:987-1001. DOI: $10.1007 / \mathrm{s} 00425-009-0888-4$.

29. Nurk S, Bankevich A, Antipov D, Gurevich A, Korobeynikov A, Lapidus A, Prjibelsky A, Pyshkin A, Sirotkin A, Sirotkin Y, Stepanauskas R, McLean J, LaskenR, Clingenpeel SR, Woyke T, Tesler G, Alekseyev MA, Pevzner PA. 2013. Assembling Genomes and Mini-metagenomes from Highly Chimeric Reads. Research in Computational Molecular Biology. Lecture Notes in Computer Science 7821: 158-170. DOI https://doi.org/10.1007/978-3-642-37195-0_13.

30. Reddemann A., Horn R. 2018. Recombination Events Involving the atp 9 Gene Are Associated with Male Sterility of CMS PET2 in Sunflower. International Journal of Molecular Sciences 19: 806. DOI:10.3390/ijms19030806.

31. Sabar M, Gagliardi D, Balk J, Leaver CJ. 2003. ORFB is a subunit of F1F(O)-ATP synthase: insight into the basis of cytoplasmic male sterility in sunflower. EMBO Reports 4:381-386. 
424

425

426

427

428

429

430

431

432

433

434

435

436

437

438

439

440

441

442

443

444

445

446

447

448

449

450

451

452

453

454

32. Siculella L, Palmer JD. 1988. Physical and gene organization of mitochondrial DNA in fertile and male sterile sunflower. CMS-associated alterations in structure and transcription of the atpA gene. Nucleic Acids Research 16:3787-3799.

33. Sloan DB, Alverson AJ, Chuckalovcak JP, Wu M, McCauley DE, Palmer JD, Taylor DR. 2012 Rapid Evolution of Enormous, Multichromosomal Genomes in Flowering Plant Mitochondria with Exceptionally High Mutation Rates. PLoS Biology 10(1): e1001241. DOI: https://doi.org/10.1371/journal.pbio.1001241.

34. Spassova M, Moneger F, Leaver CJ, Petrov P, Atanassov A, Nijkamp HJ, Hille J. 1994. Characterisation and expression of the mitochondrial genome of a new type of cytoplasmic male-sterile sunflower. Plant Molecular Biology 26: 1819-1831. DOI https://doi.org/10.1007/BF00019495.

35. Tan GF, Wang F, Zhang XY, Xiong AI. 2017. Different lengths, copies and expression levels of the mitochondrial atp6 gene in male sterile and fertile lines of carrot (Daucus carota L.). Mitochondrial DNA. Part A, DNA Mapping, Sequencing, and Analysis 1-9. DOI: $10.1080 / 24701394.2017 .1303492$.

36. Thorvaldsdóttir H, Robinson JT, Mesirov JP. 2013. Integrative Genomics Viewer (IGV): high-performance genomics data visualization and exploration. Briefings in Bioinformatics 14:178-192. DOI: 10.1093/bib/bbs017.

37. Touzet P, Meyer EH. 2014. Cytoplasmic male sterility and mitochondrial metabolism in plants. Mitochondrion 19:166-171. DOI: 10.1016/j.mito.2014.04.009.

38. Triska M, Solovyov V, Baranova A, Kel A, Tatarinova T. 2017. Nucleotide patterns aiding in prediction of eukaryotic promoters. PLoS ONE 12(11): e0187243. https://doi.org/10.1371/journal.pone.0187243

39. Whelan EDP. 1980. A new source of cytoplasmic male sterility in sunflower. Euphytica 29:33-46.

40. Yang JH, Huai Y, Zhang MF. 2009. Mitochondrial atpA gene is altered in a new orf220type cytoplasmic male-sterile line of stem mustard (Brassica juncea). Molecular Biology Reports 36:273-280. DOI 10.1007/s11033-007-9176-1.

41. Yang J, Liu1 G, Zhao1 N, Chen S, Liu D, Ma1 W, Hu Z, Zhan M. 2015. Comparative mitochondrial genome analysis reveals the evolutionary rearrangement mechanism in Brassica. Plant Biology 18:527-536. DOI: 10.1111/plb.12414. 


\section{Table $\mathbf{1}$ (on next page)}

The primers sets used for HA89 (PET2) genome reorganizations validation and gene expression analysis.

All PCR reactions were held for HA89 fertile and CMS (PET2) lines. For simplicity primers were named according to their position in the HA89 fertile genome. The ' $F$ ' and ' $R$ ' letters denote the PCR strand orientation - forward (plus) and reverse (minus), respectively. 


\begin{tabular}{|c|c|c|c|c|c|}
\hline The purpose & $\begin{array}{l}\text { Primer } \\
\text { name }\end{array}$ & Primer sequence (5'-3') & Line & $\begin{array}{c}\text { The } \\
\text { expected } \\
\text { amplicon } \\
\text { size (kbp) }\end{array}$ & $\begin{array}{c}\text { The real } \\
\text { amplicon } \\
\text { size } \\
\text { (kbp) }\end{array}$ \\
\hline \multirow{6}{*}{$\begin{array}{l}\text { Validation sub- } \\
\text { genome structure } \\
153,5 \mathrm{~kb} \text { circle }\end{array}$} & \multirow{2}{*}{$189837 \mathrm{~F}$} & \multirow{2}{*}{ CGTGAAGCCGGGATGGTATT } & Fertile & 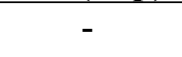 & 0.9 \\
\hline & & & PET1 & - & 0.9 \\
\hline & $37192 \mathrm{R}$ & CAAGTGATCCCCCATCCAGG & PET2 & 0.9 & 0.9 \\
\hline & \multirow{3}{*}{$\begin{array}{l}189660 \mathrm{~F} \\
37954 \mathrm{R}\end{array}$} & \multirow{2}{*}{ AGGAGTGAGATGGACGCTCT } & Fertile & - & 1.8 \\
\hline & & & PET1 & - & 1.8 \\
\hline & & AAGIGIIGCACCCCCTIGAA & PET2 & 1.8 & 1.8 \\
\hline \multirow{2}{*}{$\begin{array}{l}\text { The analysis of } \\
\text { orf645 expression }\end{array}$} & orf645F & GCCTTCCACCTCTCGTTTGA & Fertile & - & - \\
\hline & orf645R & TCCGAAAGCCGGCCTAAAAT & PET2 & 0.162 & 0.162 \\
\hline \multirow{2}{*}{$\begin{array}{c}\text { The analysis of } \\
\text { orf } 2565 \text { expression }\end{array}$} & orf $2565 \mathrm{~F}$ & TCAATCCATGTGTTCTCGCT & Fertile & - & - \\
\hline & $\operatorname{orf} 2565 \mathrm{R}$ & CGGAAAGAACAGGTCTCGGT & PET2 & 0.147 & 0.147 \\
\hline \multirow{6}{*}{$\begin{array}{l}\text { The analysis of } \\
\text { atp6 906/1056 bp } \\
\text { transcripts }\end{array}$} & \multirow{3}{*}{$\begin{array}{l}\text { atp6F } \\
\text { atp6R }\end{array}$} & \multirow{3}{*}{$\begin{array}{l}\text { AGAACTGTAACTGACAACGC } \\
\text { ACCTGAGTCCGAGTCTGCATC }\end{array}$} & Fertile & 0.106 & 0.106 \\
\hline & & & PET1 & 0.106 & 0.106 \\
\hline & & & PET2 & 0.106 & 0.106 \\
\hline & \multirow{3}{*}{$\begin{array}{l}\text { atp6- } \\
\text { 1056F } \\
\text { atp6R }\end{array}$} & \multirow{3}{*}{$\begin{array}{c}\text { TCCCATGCCTTTCTTGGTCG } \\
-\|-\end{array}$} & Fertile & - & 0.28 \\
\hline & & & PET1 & - & 0.28 \\
\hline & & & PET2 & 0.28 & 0.28 \\
\hline \multirow{6}{*}{$\begin{array}{l}\text { The analysis of } \\
\text { atp9 261/300 bp } \\
\text { transcripts }\end{array}$} & \multirow{3}{*}{$\begin{array}{l}\text { atp9F } \\
\text { atp9R }\end{array}$} & \multirow{2}{*}{ CATTGGGGCAAACGATGCAA } & Fertile & 0.107 & 0.107 \\
\hline & & & PET1 & 0.107 & 0.107 \\
\hline & & CCTCGATTCATTCCGTGGCT & PET2 & 0.107 & 0.107 \\
\hline & atp9F & $-\|-$ & Fertile & - & 0.233 \\
\hline & \multirow[t]{2}{*}{ atp9.300R } & TGAAAAAGAAAAAGCGTGAG & PET1 & - & 0.233 \\
\hline & & GAGA & PET2 & - & 0.233 \\
\hline \multirow{2}{*}{$\begin{array}{l}\text { The analysis of } \\
\text { atpl expression }\end{array}$} & atp1F & CCCATGGCACAGCCAGAATA & Fertile & 0.14 & 0.14 \\
\hline & atp1R & CAGAAACGCTCAACTGTGGC & PET2 & 0.14 & 0.14 \\
\hline \multirow{2}{*}{$\begin{array}{l}\text { The analysis of } \\
\text { orf285 expression }\end{array}$} & orf $285 F$ & TCCCATCATGACCTACCCGT & Fertile & - & - \\
\hline & atp9.300R & $-\|-$ & PET2 & 0.243 & 0.243 \\
\hline Sanger & $274655 \mathrm{~F}$ & $-\|-$ & Fertile & - & - \\
\hline resequencing the 5 & Pet2-seqR & GAAGGAACGAGACAGCACCA & PET2 & 0.7 & 0.7 \\
\hline and 3 ' ends of & Pet2-seqF & AGGGAGAGGGACGAAGTGAC & Fertile & - & - \\
\hline 5050 bp insertion & 275503R & TAACCGCTGCAAGAGTGAGG & PET2 & 0.7 & 0.7 \\
\hline Detection the 5, & $35202 \mathrm{~F}$ & AGCTCTCCCCATCGGTAGTT & Fertile & - & - \\
\hline
\end{tabular}




$\begin{array}{cccccc}\text { and 3' end of } & \text { 271194R } & \text { GGTCATCAGTTCGAGTGGCA } & \text { PET2 } & 2.5 & 2.5 \\ \text { 5885 bp insertion } & \text { Pet2-insF } & \text { AGGAAAAGACCCAACAGGCA } & \text { Fertile } & - & - \\ & 194675 \mathrm{R} & \text { TAGCTCTTCCGGAGCACTCT } & \text { PET2 } & 2.7 & 2.7\end{array}$




\section{Table 2 (on next page)}

Variation sites in mitochondrial DNA of HA89 CMS lines PET1 and PET2.

Nucleotide positions are specified according to fertile line mtDNA. IGR - an intergenic region. In case of indels, the deletions are indicated as "_" and the inserted nucleotide are in bold. 


\begin{tabular}{|c|c|c|c|c|c|}
\hline Position & Type & Fertile & PET1 & PET2 & Localization \\
\hline 3031 & SSR & G5 & & G6 & IGR nad2-ccmC \\
\hline 3107 & SSR & $\mathrm{T} 5$ & & T6 & IGR nad2-ccmC \\
\hline $3275-3276$ & INDEL & TA & & TTTA & IGR nad2-ccmC \\
\hline $3281-3281$ & INDEL & AT & & ATT & IGR nad2-ccmC \\
\hline 4715 & SSR & $\mathrm{T} 8$ & & T9 & IGR nad2-ccmC \\
\hline 6207 & SSR & A8 & A7 & A7 & IGR nad2-ccmC \\
\hline 6660 & SNP & A & & $\mathbf{G}$ & IGR nad2-ccmC \\
\hline 7404 & SSR & G10 & & G9 & IGR nad2-ccmC \\
\hline 7919 & INDEL & A & & - & IGR nad2-ccmC \\
\hline 9796 & SNP & $\mathrm{T}$ & & $\mathbf{C}$ & IGR nad2-ccmC \\
\hline 10467 & SNP & A & & $\mathbf{C}$ & IGR nad2-ccmC \\
\hline 10924 & SNP & A & & $\mathbf{C}$ & IGR nad2-ccmC \\
\hline 12314 & SNP & $\mathrm{T}$ & & $\mathbf{C}$ & IGR nad2-ccmC \\
\hline 19594 & SNP & G & & $\mathbf{A}$ & IGR $c c m C-a t p 4$ \\
\hline 23917 & SNP & G & & $\mathbf{T}$ & IGR $c c m C-a t p 4$ \\
\hline 31803 & SNP & A & & $\mathbf{C}$ & IGR nad4L-orf 259 \\
\hline 34099 & SNP & A & & $\mathbf{C}$ & IGR nad4L-orf 259 \\
\hline $34135-34136$ & INDEL & AT & & ATGT & IGR nad4L-orf 259 \\
\hline 34162 & SNP & $\mathrm{T}$ & & $\mathbf{C}$ & IGR nad4L-orf 259 \\
\hline 35031 & SNP & $\mathrm{C}$ & & $\mathbf{A}$ & IGR nad4L-orf 259 \\
\hline 35114 & SNP & $\mathrm{C}$ & & $\mathbf{A}$ & IGR nad4L-orf 259 \\
\hline 35478 & SNP & $\mathrm{T}$ & & $\mathbf{C}$ & IGR nad4L-orf 259 \\
\hline 35511 & SNP & G & & $\mathbf{A}$ & IGR nad4L-orf 259 \\
\hline 35596 & SNP & $\mathrm{G}$ & & $\mathbf{C}$ & IGR nad4L-orf 259 \\
\hline 36360 & SNP & $\mathrm{T}$ & $\mathbf{G}$ & - & orf259 Asp251Glu \\
\hline 42295 & SNP & $\mathrm{C}$ & & $\mathbf{A}$ & IGR coxIII-rpl5 \\
\hline 46039 & INDEL & A & - & & IGR rpl5-nad4 \\
\hline 49272 & SSR & $\mathrm{C} 11$ & C9 & $\mathrm{C10}$ & IGR nad4-ccmB \\
\hline 50856 & SNP & $\mathrm{C}$ & & $\mathbf{A}$ & IGR nad4-ccmB \\
\hline 51678 & SSR & G10 & G9 & G9 & IGR nad4-ccmB \\
\hline 62360 & SNP & $\mathrm{T}$ & & $\mathbf{G}$ & IGR nad4-ccmB \\
\hline 62403 & SNP & G & & $\mathbf{A}$ & IGR nad4-ccmB \\
\hline $63433-63434$ & INDEL & $\mathrm{TC}$ & & $\mathrm{TCC}$ & IGR nad4-ccmB \\
\hline $71497-71498$ & INDEL & GT & & GGGGCT & IGR rpl10-nad1 \\
\hline
\end{tabular}




\begin{tabular}{|c|c|c|c|c|c|}
\hline 75332 & SNP & A & $\mathbf{C}$ & $\mathbf{C}$ & IGR rpl10-nad1 \\
\hline 91105 & SNP & $\mathrm{G}$ & & $\mathbf{T}$ & IGR rpl10-nad1 \\
\hline 91106 & SNP & A & & $\mathbf{C}$ & IGR rpl10-nad1 \\
\hline 105474 & SSR & $\mathrm{T} 35$ & & T25 & IGR nad1-coxI \\
\hline 108200 & SNP & $\mathrm{T}$ & & $\mathbf{G}$ & IGR coxI-rps 11 \\
\hline 115915 & SNP & $\mathrm{T}$ & & $\mathbf{G}$ & IGR atp9-rps4 \\
\hline 116777 & SNP & $\mathrm{G}$ & $\mathbf{T}$ & $\mathbf{G}$ & IGR atp9-rps4 \\
\hline 119331 & SNP & $\mathrm{G}$ & & $\mathbf{A}$ & IGR atp9-rps4 \\
\hline $121108-121109$ & INDEL & $\mathrm{CC}$ & & CTTC & IGR atp9-rps4 \\
\hline 122990 & SNP & A & & $\mathbf{C}$ & rps4 (synonymous) \\
\hline 133546 & SNP & $\mathrm{T}$ & & $\mathbf{A}$ & IGR rrn26-rrn5 \\
\hline $133547-133548$ & INDEL & $\mathrm{AT}$ & & $\mathrm{A} \mathbf{G G}$ & IGR rrn26-rrn5 \\
\hline 133548 & SNP & $\mathrm{T}$ & & $\mathbf{G}$ & IGR rrn26-rrn5 \\
\hline 133549 & SNP & A & & $\mathbf{C}$ & IGR rrn26-rrn5 \\
\hline 156213 & SNP & $\mathrm{C}$ & & $\mathbf{A}$ & IGR rps 13-nad6 \\
\hline $156621-156622$ & INDEL & $\mathrm{CC}$ & & CCTAC & IGR rps 13-nad6 \\
\hline 157459 & SNP & $\mathrm{T}$ & & $\mathbf{G}$ & IGR rps 13-nad6 \\
\hline 169028 & SNP & G & $\mathbf{T}$ & & nad6 (Ser232Tyr) \\
\hline 170185 & SSR & $\mathrm{T} 14$ & T12 & T12 & IGR nad6-ymf16 \\
\hline $174932-174933$ & INDEL & $\mathrm{AC}$ & & $\begin{array}{c}\text { ACTCGACTGAA } \\
\text { AGGAAAGGTA } \\
\text { CGAAGTGGC }\end{array}$ & IGR nad6-ymf16 \\
\hline 175179 & SNP & $\mathrm{G}$ & & $\mathbf{T}$ & IGR nad6-ymf16 \\
\hline 178406 & SSR & T9 & T8 & & $y m f 16$ intron \\
\hline 184739 & SSR & A10 & A11 & & IGR ymf16-cob \\
\hline 188363 & SSR & $\mathrm{T} 11$ & T10 & T10 & $c o b$ intron \\
\hline 189980 & SNP & G & & $\mathbf{T}$ & IGR $c o b-c c m F c$ \\
\hline 195008 & SNP & $\mathrm{G}$ & & $\mathbf{T}$ & IGR $c o b-c c m F c$ \\
\hline 195015 & SNP & $\mathrm{C}$ & & $\mathbf{A}$ & IGR $c o b-c c m F c$ \\
\hline 200174 & SNP & G & & $\mathbf{A}$ & $c c m f C$ intron \\
\hline 200515 & SNP & G & & $\mathbf{A}$ & $c c m f C$ intron \\
\hline 202672 & SNP & $\mathrm{T}$ & $\mathbf{C}$ & & IGR orf873-atp1 \\
\hline 204990 & SNP & $\mathrm{C}$ & & $\mathbf{A}$ & IGR atpl-ccmFn \\
\hline 204846-204847 & INDEL & $\mathrm{AA}$ & & ATA & IGR atpl-ccmFn \\
\hline 207965 & SSR & G10 & & G12 & IGR atpl-ccmFn \\
\hline
\end{tabular}




\begin{tabular}{|c|c|c|c|c|c|}
\hline 209335-36 & INDEL & $\mathrm{AA}$ & - & & IGR atpl-ccmFn \\
\hline 209458 & SNP & $\mathrm{G}$ & & $\mathbf{A}$ & IGR atpl-ccmFn \\
\hline 212638 & SSR & $\mathrm{C} 9$ & & $\mathrm{C12}$ & IGR atp 1-ccmFn \\
\hline 215916 & SNP & $\mathrm{C}$ & & $\mathbf{T}$ & IGR $c c m F n-n a d 7$ \\
\hline 223917 & SNP & A & & $\mathbf{C}$ & IGR nad7-rps3 \\
\hline $3925-223926$ & INDEL & GA & & GAA & IGR nad7-rps3 \\
\hline $26977-226978$ & INDEL & $\mathrm{AC}$ & & ACGTTGTTTTC & IGR nad7-rps3 \\
\hline 230112 & SNP & A & $\mathbf{C}$ & & rpl16 (Lys32Gln) \\
\hline 232826 & SNP & G & & $\mathbf{T}$ & IGR rpl16-matR \\
\hline 239880 & SNP & G & & $\mathbf{A}$ & IGR rpl16-matR \\
\hline 239988 & SNP & A & & $\mathrm{C}$ & IGR rpl16-matR \\
\hline 241035 & SNP & G & & $\mathbf{A}$ & IGR rpl16-matR \\
\hline 241475 & SNP & A & & $\mathbf{C}$ & IGR rpl16-matR \\
\hline 246053 & SNP & $\mathrm{C}$ & & $\mathbf{T}$ & IGR rpl16-matR \\
\hline 248266 & SSR & A14 & A10 & A9 & IGR rpl16-matR \\
\hline 249347 & SSR & $\mathrm{T} 8$ & & T9 & IGR rpl16-matR \\
\hline 249361 & SNP & $\mathrm{C}$ & & $\mathbf{A}$ & IGR rpl16-matR \\
\hline 260901 & SNP & G & & $\mathbf{T}$ & IGR nad9-atp6 \\
\hline 262080 & SNP & G & & $\mathbf{A}$ & IGR nad9-atp6 \\
\hline 269062 & SNP & G & $\mathbf{C}$ & $\mathbf{C}$ & IGR nad9-atp6 \\
\hline 269134 & SNP & A & & $\mathrm{C}$ & atp6 (synonymous) \\
\hline 270676 & SNP & G & & $\mathbf{T}$ & IGR atp6-coxII \\
\hline 273344 & SNP & $\mathrm{C}$ & & $\mathbf{A}$ & IGR atp6-coxII \\
\hline 276834 & SNP & $\mathrm{T}$ & & $\mathbf{G}$ & IGR atp6-coxII \\
\hline
\end{tabular}


Figure 1

Graphical mitochondrial genome maps of HA89 fertile line. 
Figure 2

Graphical mitochondrial genome map of HA89(PET1) line 
Figure 3

Graphical mitochondrial genome map of HA89(PET2) line

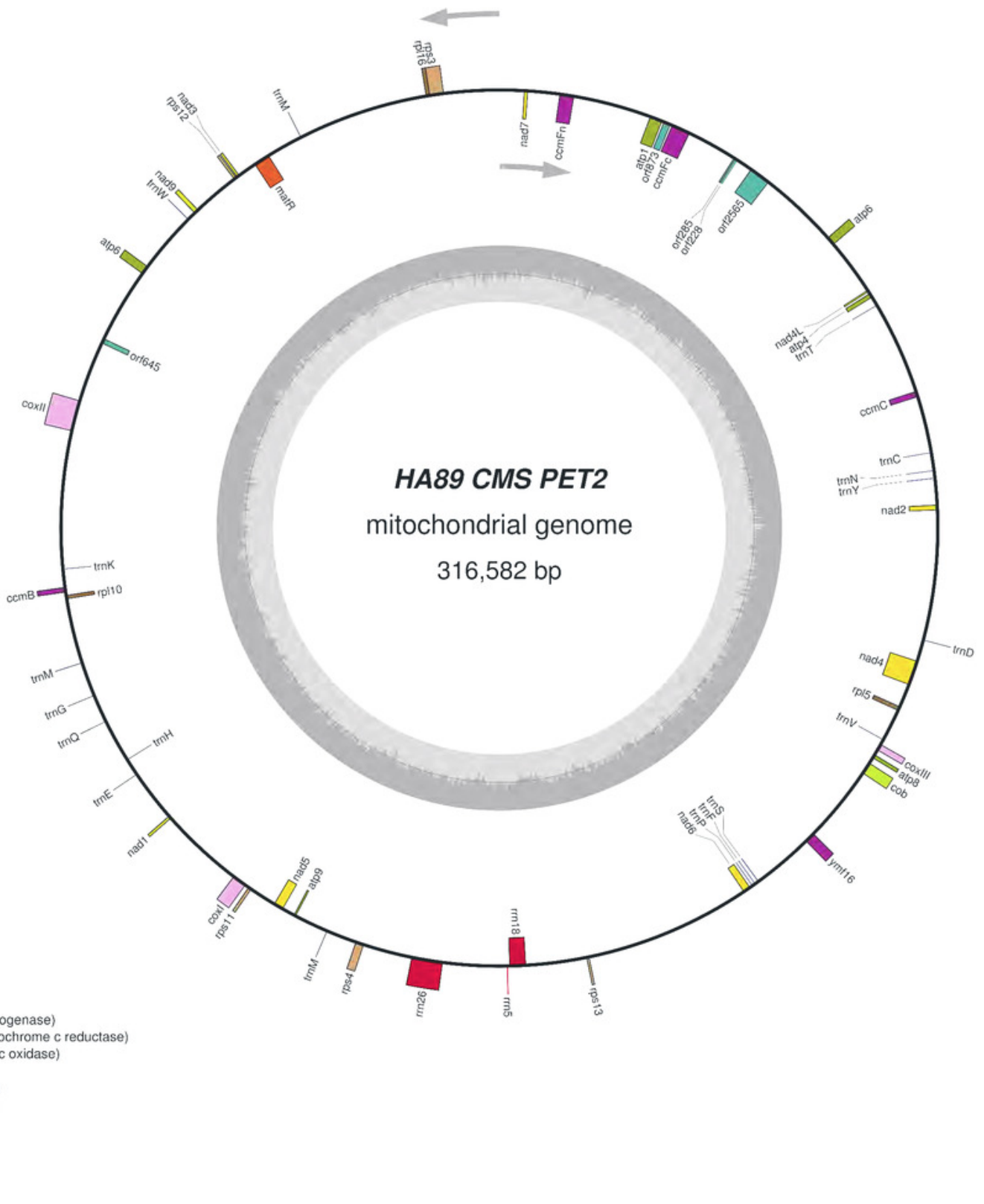

